\title{
Primera cita de la especie invasora Caulerpa cylindracea Sonder (Caulerpaceae, Chlorophyta) en las islas Chafarinas (África del Norte)
}

Julio Peñas de Giles ${ }^{1}$, Jesús del Río Sánchez ${ }^{2}$, Luís Sánchez-Tocino ${ }^{3}$ \& Antonio de la Linde Rubio ${ }^{4}$

1 Unidad de Conservación Vegetal, Dpto. Botánica, Universidad de Granada. Granada, España.

2 Dpto. de Geodiversidad y Biodiversidad, Delegación Territorial de la Consejería de Agricultura, Ganadería, Pesca y Desarrollo Sostenible. Junta de Andalucía. Granada, España.

${ }^{3}$ Dpto. Zoología, Universidad de Granada. Granada, España.

${ }^{4}$ Agencia de Medio Ambiente y Agua de Andalucía. Cádiz, España.

Correspondencia: Julio Peñas e-mail: jgiles@ugr.es

Recibido: 18 septiembre 2019

Aceptado: 18 marzo 2020

Publicado on-line: 13 mayo 2020

Editado por: María Altamirano Jeschke

\begin{abstract}
Resumen
Las Islas Chafarinas constituyen una importante área de biodiversidad en el Mar de Alborán (Mediterráneo occidental) que se encuentra bajo protección. Presentamos un nuevo registro de presencia de Caulerpa cylindracea para las islas, un taxón invasor agresivo y de rápida expansión introducido en el mar Mediterráneo hace unos años. Alertamos sobre una posible invasión en esta importante reserva marina.
\end{abstract}

Palabras clave: Conservación, especies invasoras, Mar de Alborán, reserva marina.

\begin{abstract}
First record of the invasive species Caulerpa cylindracea Sonder (Caulerpaceae, Chlorophyta) from Chafarinas Islands (N Africa)

The Chafarinas Islands constitute an important biodiversity area in the Alboran Sea (western Mediterranean) that is under protection. For the islands, we present a new record of Caulerpa cylindracea, an aggressive, fast-spreading invasive taxon introduced into the Mediterranean Sea a few years ago. We warn about a potential invasion in this significant marine reserve.
\end{abstract}

Key words: Alboran Sea, conservation, invasive species, marine reserve.
Las islas Chafarinas (también llamadas Zafarin o Djaferin) constituyen un pequeño archipiélago del mar de Alborán (Mediterráneo occidental), una importante ecorregión marina de interés para la conservación (Olson \& Dinerstein, 2002; Watson et al., 2014). Está formado por tres islotes volcánicos (Congreso, Isabel II y Rey; Figura 1) situados frente a la costa de Marruecos, a una distancia de $3,52 \mathrm{~km}$. Este archipiélago es una notable área marina protegida por las leyes españolas y europeas, como Refugio Nacional de Caza (desde 1982), ZEPA-Zona de Especial Protección para las Aves (desde 1989), ZEC-Zona de Especial Conservación (desde 2018) y por tanto integrante de la Red Natura 2000 (European Nature Information System, 2019), debido a la rica biodiversidad que alberga, tanto terrestre como marina, y a su ubicación biogeográfica clave, confluencia de biotas mediterránea y atlántica.

Desde el primer estudio sobre la flora marina de las islas Chafarinas (Conde, 1984), se han registrado 169 taxones específicos e infraespecíficos, incluyendo cianobacterias, macroalgas y la angios-perma marina Posidonia oceanica (L.) Delile (Altamirano, 1999; Altamirano et al., 2010; Altamirano et al., 2013; De la Rosa et al., 2013). Por otro lado, han sido citadas dos especies invasoras de algas rojas, como son Asparagopsis taxiformis (Delile) Trevisan y $A$. armata Harvey (Altamirano, 1999; Altamirano et al., 2010).

Una de las principales amenazas a la biodiversidad es la invasión de especies exóticas en áreas importantes de biodiversidad y de servicios ecosistémicos (Simberloff et al., 2013). El efecto negativo de las invasiones biológicas está aumentando, especialmente en el medio marino donde se está vinculado a la intensificación del tráfico marítimo, la acuicultura y la actividad de los acuarios (Carlton, 1989; Verlaque, 1994).

Con este trabajo se confirma por primera vez en las islas Chafarinas la presencia del alga verde invasora Caulerpa cylindracea (Figura 2), una de las invasoras más conspicuas incluida en la lista de las 100 "Peores especies exóticas invasoras" del Mediterráneo (Streftaris \& Zenetos, 2006), que afecta a escala local a la diversidad de macroalgas y de praderas marinas (Klein \& Verlaque, 2008; Gennaro \& Piazzi, 2011). En las costas noroccidentales de África se ha registrado en el lado atlántico de Ceuta (Rivera-Ingraham et al., 2010) y 

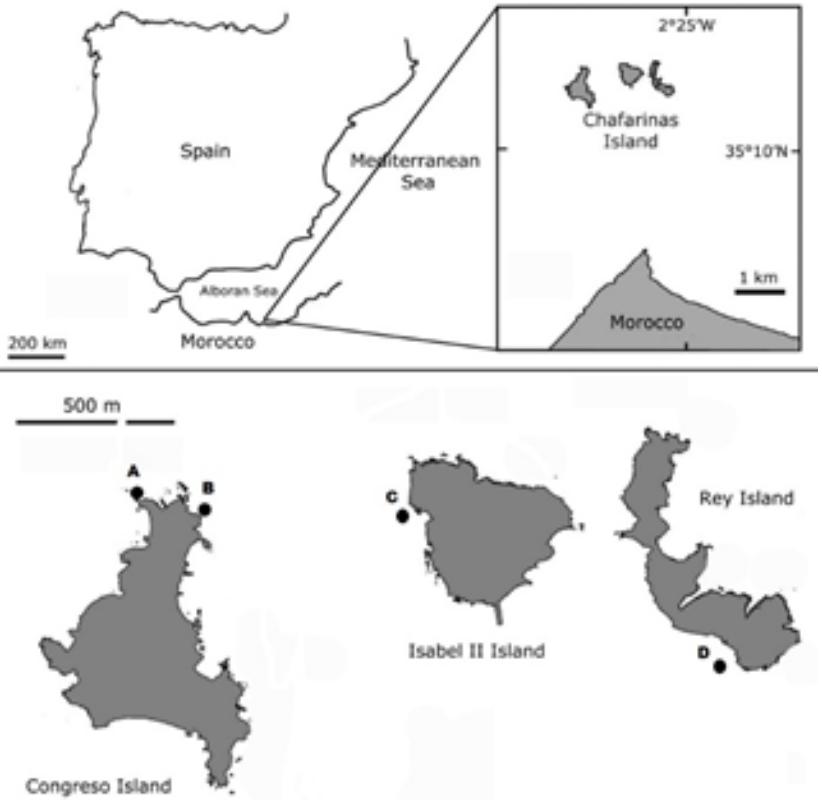

Figura 1. Ubicación del archipiélago de Chafarinas en el Mar de Alborán (Mediterráneo occidental), y las tres islas: Congreso, Isabel II y Rey. Se muestran los puntos de presencia (A-D) de Caulerpa cylindracea en 2018 y en 2019, ver Tabla 1.

Figure 1. Location of Chafarinas archipelago in Alboran Sea (western Mediterranean Sea), and the tree islands: Congreso, Isabel II, and Rey. Points of presence (A-D) of Caulerpa cylindracea in 2018 and 2019 are shown, see Table 1.

en el Golfo de Arzew (Argelia occidental; Bouiadjra et al., 2010), y se sospechaba de su presencia en las costas marroquíes (Verlaque et al., 2015).

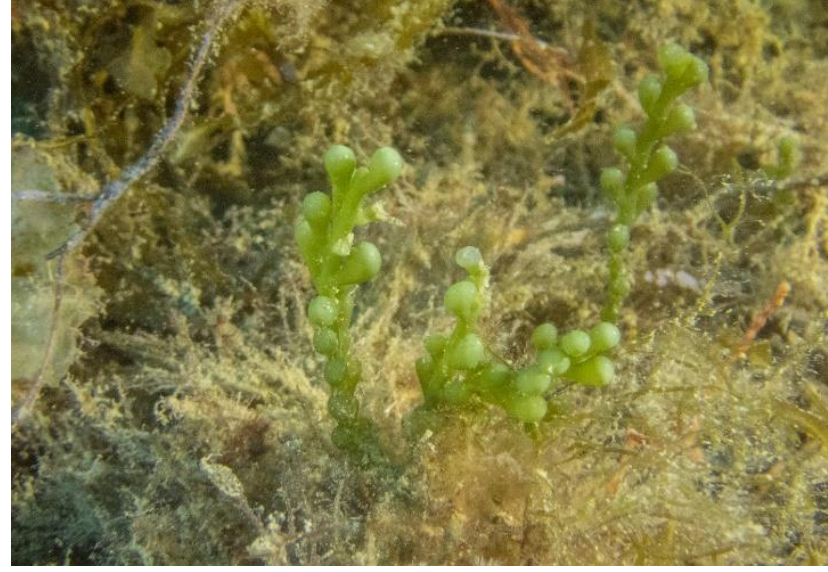

Figura 2. Caulerpa cylindracea Sonder en isla del Congreso (islas Chafarinas).

Figure 2. Caulerpa cylindracea Sonder in Congreso Island (Chafarinas Islands).

En agosto de 2018 se recogieron muestras en la isla del Congreso que fueron llevadas al laboratorio de la Estación Biológica de las islas Chafarinas para ser identificadas y preservadas; posteriormente se incluyeron en el Herbario de la Universidad de Granada (con el registro GDA-62739), y en el verano de 2019 se detectó también en las islas de Isabel II y del Rey (Tabla 1). Los talos aparecían de manera ocasional, no continua, presentando una estructura cespitosa con estolones reptantes de donde salen haces erguidos (frondes) de hasta $10 \mathrm{~cm}$ de largo. Junto con C. cylindracea, predomina Dictyopteris polypodioides (A.P. De Candolle) J.V. Lamouroux y también Padina pavonica (L.) Thivy en abundancia,

Tabla 1. Presencia de Caulerpa cylindracea Sonder en las islas Chafarinas en 2018 y 2019 . Se indican las coordenadas (las letras corresponden a los puntos de la Figura 1), batimetría y ecología donde se localizó.

Table 1. Presence of Caulerpa cylindracea Sonder in Chafarinas Islands in 2018 and 2019. The coordinates (letters correspond to the points in Figure 1), bathymetry and ecology where it was located.

Isla del Congreso $\quad$ Isla de Isabel II $\quad$ Isla del Rey

\begin{tabular}{|c|c|c|c|c|}
\hline \multirow[t]{3}{*}{$\begin{array}{c}\text { Presencias } \\
\text { en } 2018\end{array}$} & $\begin{array}{c}\text { Coordenadas } \\
\text { geográficas y } \\
\text { (fecha) }\end{array}$ & $\begin{array}{r}\mathrm{A}-35^{\circ} 11^{\prime} 00,35^{\prime \prime} \mathrm{N} \\
2^{\circ} 26^{\prime} 29,81^{\prime \prime} \mathrm{W} \\
\mathrm{B}-35^{\circ} 10^{\prime} 59,49^{\prime \prime} \mathrm{N} \\
2^{\circ} 26^{\prime} 20,56^{\prime \prime} \mathrm{W} \\
(04-09-2018)\end{array}$ & & \\
\hline & $\begin{array}{l}\text { Batimetría } \\
(\mathrm{m})\end{array}$ & $17,5-25,6$ & & \\
\hline & Ecología & $\begin{array}{c}\text { sustratos duros } \\
\text { y arenosos }\end{array}$ & & \\
\hline \multirow{3}{*}{$\begin{array}{c}\text { Presencias } \\
\text { en } 2019\end{array}$} & $\begin{array}{c}\text { Coordenadas } \\
\text { geográficas y } \\
\text { (fecha) }\end{array}$ & $\begin{array}{r}\mathrm{B}-35^{\circ} 10^{\prime} 59,49^{\prime \prime} \mathrm{N} \\
2^{\circ}-26^{\prime} 20,56^{\prime \prime} \mathrm{W} \\
(19-08-2019)\end{array}$ & $\begin{array}{c}\mathrm{C}-35^{\circ} 10^{\prime} 56,79^{\prime \prime} \mathrm{N} \\
2^{\circ} 25^{\prime} 56,87^{\prime \prime} \mathrm{W} \\
(23-08-2019)\end{array}$ & $\begin{array}{c}\mathrm{D}-35^{\circ} 10^{\prime} 38,73^{\prime \prime} \mathrm{N} \\
2025^{\prime} 13,44^{\prime \prime} \mathrm{W} \\
(24-08-2019)\end{array}$ \\
\hline & $\begin{array}{l}\text { Batimetría } \\
(\mathrm{m})\end{array}$ & $16-26$ & $14,5-19$ & 21,6 \\
\hline & Ecología & $\begin{array}{l}\text { sustratos duros } \\
\text { y arenosos }\end{array}$ & $\begin{array}{l}\text { sustratos arenosos } \\
\text { y fondos de } \\
\text { Posidonia oceánica }\end{array}$ & $\begin{array}{l}\text { sustratos duros } \\
\text { y arenosos }\end{array}$ \\
\hline
\end{tabular}


junto con algas calcáreas de los géneros Lithophyllum y Mesophyllum. En los espacios dejados por las algas, podemos observar invertebrados como los poliquetos (Polychaeta) Protula intestinum (Lamarck, 1818), P. tubularia (Montagu, 1803) y Serpula vermicularis (Linnaeus, 1767); las esponjas (Porifera) Crambe crambe (Schmidt, 1862), Sarcotragus spinosulus (Schmidt, 1862) y Sarcotragus fasciculatus (Pallas, 1766); y los equinodermos (Echinodermata) Sphaerechinus granularis (Lamarck, 1816) y Ophidiaster ophidianus (Lamarck, 1816). Caulerpa cylindracea también fue detectada en 2014 en varios lugares del Parque Natural del Estrecho (Tarifa, Punta Paloma y Guadalmesí; CMAOT, 2015), así como en múltiples localidades del Parque Natural de Cabo de Gata-Níjar (costa del SE ibérico; observ. pers.), otra importante reserva de biodiversidad marina. Es probable que pueda colonizar de forma rápida y con mayor abundancia nuevas áreas del archipiélago de las Chafarinas (durante otras inmersiones han sido detectados fragmentos de talos a la deriva). El presente trabajo advierte sobre esta amenaza potencial de $C$. cylindracea, lo cual puede ser útil para la gestión de la conservación de esta importante reserva marina del Mediterráneo occidental.

\section{Agradecimientos}

Los autores agradecen al destacamento militar de las islas Chafarinas, al personal del Organismo Autónomo de Parques Nacionales, especialmente a Javier Díaz, y a nuestros compañeros de buceo Soledad Lizana y Teodoro Pérez Guerra por su ayuda y colaboración.

\section{Bibliografía}

Altamirano, M. (1999). Nuevas citas para la flora marina del archipiélago de las islas Chafarinas. Acta Botanica Malacitana, 24, 185-233.

Altamirano, M., De la Rosa, J., Zanolla, M., SouzaEgipsy, V. \& Díaz, J. (2010). New records for the benthic marine flora of Chafarinas islands (Alboran Sea, Western Mediterranean). Acta Botanica Malacitana, 35, 165-167.

Altamirano, M., De la Rosa, J., Flagella, M. \& Zanolla, M. (2013). Contributions to the benthic marine flora of Chafarinas Islands (Alboran Sea, Western Mediterranean). Acta Botanica Malacitana, 38, 187-191.

Bouiadjra, B.B., Taleb, M.Z., Marouf, A, Benkada, M.Y. \& Riadi, H. (2010). First record of the invasive alga Caulerpa racemosa (Caulerpales, Chlorophyta) in the Gulf of Arzew (western Algeria). Aquatic Invasions, 5, 97-101.
Carlton, J.T. (1989). Man's role in changing the face of the ocean: biological invasions \& implications for conservation of near-shore environments. Conservation Biology, 3, 265-273.

Conde, F. (1984). Contribución al conocimiento de la flora algal bentónica del Mar de Alborán. Islas Chafarinas. Acta Botanica Malacitana, 9, 41-46.

Consejería de Medio Ambiente y Ordenación del Territorio (2015). Programa de Gestión Sostenible del Medio Marino Andaluz - Informe 2015. Junta de Andalucía.

De la Rosa, J., Altamirano, M. \& Zanolla, M. (2013). Checklist of benthic marine cyanoprokariota of Chafarinas Islands (Alboran Sea, Western Mediterranean). Acta Botanica Malacitana, 38, 182-186.

European Nature Information System (5 abril 2019). Islas Chafarinas. Site Natura 2000 (ES6300001). https://eunis.eea.europa.eu/sites/ES6300001

Gennaro, P. \& Piazzi, L. (2011). Synergism between two anthropic impacts: Caulerpa racemosa var. cylindracea invasion and seawater nutrient enrichment. Marine Ecology Progress Series, 427, 59-70.

Klein, J. \& Verlaque, M. (2008). The Caulerpa racemosa invasion: a critical review. Marine Pollution Bulletin, 56, 205-225.

Olson, D.M. \& Dinerstein, E. (2002). The Global 200: Priority ecoregions for global conservation. Annals of the Missouri Botanical Garden, 89, 125-126.

Rivera-Ingraham, G.A., García-Gómez, J.C. \& Espinosa, F. (2010). Presence of Caulerpa racemosa (Forsskål) J. Agardh in Ceuta (Northern Africa, Gibraltar Area). Biological Invasions, 12(6), 1465-1466.

Simberloff, D., Martin, J.L., Genovesi, P. \& al. (2013). Impacts of biological invasions: what's what and the way forward. Trends in Ecology and Evolution, 28, 58-66.

Streftaris, N. \& Zenetos, A. (2006). Alien Marine Species in the Mediterranean - the 100 'Worst Invasives' and their Impact. Mediterranean Marine Science, 7, 87-118.

Verlaque, M. (1994). Inventaire des plantes introduites en Méditerranée: origines et répercussions sur l'environnement et les activités humaines. Oceanologica Acta, 17, 1-23.

Verlaque, M., Ruitton, S., Mineur F. \& Boudouresque, C.F. (2015). CIESM Atlas of Exotic Species in the Mediterranean. Volume 4. Macrophytes.

Watson, J.E.M, Dudley, N., Segan, D.B. \& Hockings, M. (2014). The performance and potential of protected areas. Nature, 515, 67-7. 